\title{
Le vagabondage ou la flânerie subversive chez Isabelle Eberhardt
}

Amira Ben Rejeb, Université du Québec à Chicoutimi

Dans Les règles de l'art du sociologue français Pierre Bourdieu, le champ littéraire et artistique du dix-neuvième siècle connaît un éclatement qui mérite d'être étudié. Selon Bourdieu, la place dominante d'un écrivain dans le champ littéraire permettrait à celui-ci de jouir d'une plus grande renommée et à son œuvre d'être reconnue comme une œuvre littéraire consacrée. Quant aux écrivains restés en marge, leurs noms et leurs œuvres passent vite aux oubliettes et ces dernières ne sont guère publiées par les grands éditeurs de l'époque. Étudier une auteure comme Isabelle Eberhardt, restée longtemps méconnue, considérée souvent comme la Mata Hari de la littérature, équivaut aujourd'hui à s'intéresser aux « marginaux » du champ littéraire français au dix-neuvième siècle. Suisse d'origine russe, Eberhardt détenait aussi la nationalité française et était inspirée par des auteurs français, dont Pierre Loti. Elle est considérée comme une transfuge puisqu'elle se rend en Algérie à l'âge de vingt ans et décide d'y vivre en se rangeant parfois du côté de la figure du colonisé. Elle publie la majeure partie de son œuvre dans les journaux d'Alger comme « Al Akhbar » et est accusée d'espionnage tant par les Algériens que par les Français. Elle reste dans un entredeux infernal toute sa vie durant, avant d'être emportée dans le débordement de l'oued à Aïn Sefra, en Algérie. Partie trop tôt, à vingt-sept ans, elle laisse derrière elle une œuvre importante entre fiction et récits de voyages ; à cet âge, Gustave Flaubert, auteur consacré du champ littéraire français, était encore à sa première version de L'éducation sentimentale, qu'il a jugée non publiable. Certains diront que la mort d'Eberhardt a été aussi fascinante que sa vie puisqu'elle se noie dans la boue du désert.

On l'a souvent assimilée à Arthur Rimbaud, certains sont même allés jusqu'à dire qu'elle était sa fille illégitime. Ces deux créateurs de génie partagent l'attrait du désert symbolisant l'infini, l'abolition des limites, la liberté totale et l'abandon de soi, mais aussi un attrait pour l'anarchisme, à l'origine de cette quête de liberté qui les rapproche (voir Shindler). D'après les critiques d'Eberhardt, l'auteure dite nomade reçoit durant son enfance une éducation libertaire de la part de son tuteur légal, Alexandre Trophimowsky, qui est un disciple de Mikhaïl Bakounine. Ce dernier est un philosophe russe révolutionnaire, connu pour être le théoricien de l'anarchisme, ayant particulièrement critiqué le rôle de l'État. Sous la tutelle de Trophimowsky, Eberhardt est traitée de la même façon que ses deux frères, en marge des codes genrés, et c'est probablement cette éducation loin des normes traditionnelles 
qui explique bon nombre de ses aspirations et de ses principes libertaires peu communs parmi les femmes de son époque (voir Chaulet Achour). Cet anarchisme qui teint les idées de son père l'ont d'ailleurs beaucoup influencée, et c'est ce qui sera étudié dans cet article, en rapport avec son vagabondage, mode de vie choisi par Eberhardt. L'auteure a passé sa vie d'adulte à voyager entre l'Europe et l'Afrique, faisant une rupture pleinement assumée avec la première et vouant à la deuxième un intérêt immense et un amour qui ne s'interrompra qu'à sa mort.

Dans les travaux sur Eberhardt, on met souvent l'accent sur son nomadisme, sa condamnation de l'Occident et ses relations rapprochées avec les Maghrébins qu'elle a pu connaître lors de son passage sur l'autre rive de la Méditerranée. Au début des années 1990, Laura Rice et Ali Behdad jettent la lumière sur la manière dont Eberhardt se positionne par rapport à la question de la colonisation et du genre, jouant entre les limites de ces frontières normatives. En 1993, Hédi Abdel-Jaouad met l'accent sur l'importance du bilinguisme d'Eberhardt et son expression à travers ses œuvres de fiction. Par la suite, des critiques comme Sidonie Smith (2001) et Michelle Chilcoat (2004) s'attardent sur les tendances misogynes des écrits d'Eberhardt. Dans des travaux académiques plus récents comme ceux de Dunlaith Bird (2012), d'Irmagard Scharold (2013) et de Linda Chouiten (2014), la vie de l'auteure reste le point d'ancrage, même si quelques aspects de sa fiction sont également discutés. Le personnage d'Eberhardt reste la fascination première des critiques puisqu'une femme libre au dix-neuvième siècle vivant pleinement sa passion pour l'Orient et brandissant son audace en tous lieux, reste extrêmement fascinante. Toutefois, l'étude de concepts révolutionnés par une auteure en marge d'une histoire à écrire permet de comprendre sa philosophie et les connections qu'elle établit entre ses différentes sources d'apprentissage : l'éducation, le voyage et l'ouverture à l'autre. Cet article propose d'examiner comment le vagabondage d'Eberhardt en tant que pensée et en tant que mode de vie est une subversion se différenciant du concept de la flânerie qui connaît le succès à Paris, dans des cadres bien définis. Il s'appuiera sur l'ouvrage de Catherine Nesci, Le flâneur et les flâneuses : les femmes et la ville à l'époque romantique, afin de mieux percevoir la nuance entre la flânerie en ville et le vagabondage d'Eberhardt. L'Orient, cet éternel espace historique sans âge et sans frontières fixes, qui semble être pour l'auteure marginale le lieu par excellence de la lenteur, constitue-t-il un tremplin à l'action de vagabonder qui est en soi un acte de rébellion, surtout pour une femme au dix-neuvième siècle ? 


\section{Réinventer la flânerie}

En réinventant les codes du genre et des espaces, Eberhardt réinvente aussi les concepts, dont le plus éminent dans ses écrits et dans sa vie est la flânerie ou, comme elle l'appelle, le vagabondage. Eberhardt fait l'apologie du vagabondage dans ses notes et récits de voyage et consacre une de ces nouvelles à la mise en définition de la flânerie. Mais avant d'étudier ce qu'Eberhardt, à travers sa nouvelle intitulée «Chemineau », apporte à l'idée de la flânerie au dix-neuvième siècle, il faut faire une comparaison entre le terme qu'elle choisit, « vagabondage », et le terme plus connu parmi les écrivains, «flânerie ». La flânerie et le vagabondage partagent un point commun qui est celui de l'errance. Mais, La flânerie a un cadre bien précis, la ville de Paris (voir Nesci). Quant au terme «vagabondage », il comporte une prise de position par rapport au reste de la société. Un vagabond est un rebelle pour qui les lois de la société ne s'appliquent pas, tandis qu'un flâneur est un écrivain ou un artiste qui se laisse conduire par ses pensées dans le cadre de la ville : «Être seul, être pauvre de besoin, être ignoré, étranger et chez soi partout, et marcher, solitaire et grand à la conquête du monde [...]. J'étais sortie et j'avais erré, en rêvant, dans le dédale des rues arabes, où la vie finit avec le jour » (Ebernhardt, dans Delacour et Huleu 27 ; 33).

Nesci stipule qu'il y a eu une seule forme de flâneurs, mais que les flâneuses peuvent être fort différentes et introduisent de nouvelles manières d'habiter l'espace au dix-neuvième siècle. Ces écrivain.es s'adonnent à la flânerie et se travestissent volontiers comme des bohémien.nes ou des parias, ce qui incite à voir dans Eberhardt l'illustration parfaite de cette flâneuse du désert, qui quitte l'Europe et s'établit en Algérie jusqu'à sa mort. Eberhardt fait l'éloge de la flânerie ou ce qu'elle appelle le vagabondage: «Le vagabondage, c'est l'affranchissement, et la vie le long des routes, c'est la liberté. Rompre un jour bravement toutes les entraves dont la vie moderne et la faiblesse de notre cœur, sous prétexte de liberté, ont chargé notre geste, s'armer du bâton et de la besace symboliques, et s'en aller !» (27). La vie moderne est celle de l'Europe, à laquelle elle assigne une teinte grise ; le tableau de cette vie moderne semble être restreint aux habits et aux convenances dans lesquels les femmes sont confinées. Dans ses ébauches sur le vagabondage, Eberhardt écrit : «Un droit que bien peu d'intellectuels se soucient de revendiquer, c'est le droit à l'errance, au vagabondage. Et pourtant, le vagabondage, c'est l'affranchissement, et la vie le long des routes, c'est la liberté. [...] Être seul, être pauvre de besoins, être ignoré, étranger et chez soi partout, et marcher, solitaire et grand à la conquête du monde » (27). Éprise d'aventures et de voyages, Eberhardt illustre son désir de liberté et de vagabondage dans l'image du chemineau : «Le chemineau solide, assis sur le bord de la route, et qui contemple l'horizon libre, ouvert devant 
lui, n'est-il pas le maître absolu des terres, des eaux et même des cieux ? Quel châtelain peut rivaliser avec lui en puissance et en opulence ? Aucun servage n'avilit son allure, aucun labeur ne courbe son échine vers la terre qu'il possède et qui se donne à lui, toute, en bonté et en beauté » (27). Le vocabulaire du matériel et de l'économique entre en jeu pour délimiter ce qui relève du désintéressement et ce qui touche à l'asservissement économique avilissant pour l'âme et l'esprit.

La flânerie illustrée dans l'image du chemineau qui ne connaît point de limites, exprime une résistance aux frontières et une opposition à la loi économique du travail asservissant. Pour Eberhardt, «Le besoin peureux d'immobilité, ressemble à la résignation inconsciente de la bête, que la servitude abrutit, et qui tend le cou vers le harnais » (28). Cette métaphore porte en elle l'une des caractéristiques essentielles du vagabondage ou de la flânerie : défier les limites du temps et de l'espace et se laisser aller aux hasards de la route pour conquérir sa liberté. Ainsi, le vagabondage est la rébellion contre les lois économiques, l'asservissement, le travail qui courbe l'échine et avilit l'esprit. Cette idée est le fondement de l'Art pour l'Art. Dans cette perspective, Noam Chomsky dans Instinct de Liberté: anarchisme et socialisme écrit :

Un anarchiste logique doit s'opposer à propriété privée des moyens de production et au salariat, qui sont les composantes du capitalisme parce qu'ils sont incompatibles avec le principe voulant que le travail soit libre et sous contrôle du producteur. [...] La condition préalable est l'abolition du capital et du travail salarié en tant que catégories sociales. [...] L'anarchiste logique sera socialiste, mais d'une façon particulière. Il s'opposera [entre autres] au travail aliéné et spécialisé. (27-34)

Au dix-neuvième siècle, l'Europe et la France en particulier connaissent une tension entre la logique de l'art et la logique de l'économie. Bourdieu revient d'ailleurs sur ces deux champs antithétiques qui fonctionnent par des nomos contradictoires mais liés entre eux, le champ littéraire et artistique défini par opposition aux champs politique et économique. La rupture qui se fait sentir entre la logique qui régit le travail, l'activité rémunérée, et la flânerie, le droit à l'errance, activité philosophique, intellectuelle, émane de l'opposition entre le beau et le rentable. En rupture avec l'Europe, Eberhardt rompt avec les lois qui la régissent. Le goût pour l'Orient est le goût d'une artiste en marge des champs sociaux, qui retrouve dans cet espace le lieu de tous les commencements ou recommencements.

Dans la nouvelle «Yasmina », les thèmes du nomadisme, du voyage, du désinvestissement des attaches sociales imposées s'opposent aux valeurs, aux couleurs et aux cadres de la vie moderne de la société occidentale industrialisée. Eberhardt exprime 
clairement son dégoût pour la mentalité occidentale de la fin du dix-neuvième siècle en même temps qu'elle déclare dans ses œuvres, ses notes de voyages et ses articles publiés un amour infini pour l'Algérie et le désir profond de ne faire qu'une avec cette terre : «Sous quel ciel et dans quelle terre reposerai-je au jour fixé par mon destin ? Mystère... Et cependant, je voudrais que ma dépouille fût mise dans la terre rouge de ce cimetière de la blanche Annaba [...], ou bien, alors, n'importe où, dans le sable brulé du désert, loin des banalités profanatrices de l'Occident envahisseur » (t. 1, Récits, journaliers et notes, 19).

Cependant, le cadre n'est pas le seul outil permettant cette escapade hors du temps, c'est aussi et surtout la langue qui permet l'attardement dans le présent; le métissage linguistique avec l'arabe pourrait être cette composante qui donne au texte d'Eberhardt l'aspect « flâneur », lent, sans attache au futur. En effet, contrairement aux langues indo-européennes, qui privilégient la situation sur la flèche du temps, les langues sémitiques, dont l'arabe, privilégient l'état accompli ou non. D'ailleurs, en algèbre par exemple, les Orientaux ont inventé le chiffre zéro et découvert l'abstraction, ce « $\mathrm{x} »$ qui signifie quelque chose mais rien en particulier, valeur muable indéfinie, vide qui se réclame comme une chose existant en soi, pleine et remplie de son propre appel à penser l'infini, que Omar Khayyam a nommé «Chay » ce «quelque chose », ce néant qui n'est pas matière à remplir. Dans ses écrits, Eberhardt emploie profusément le mot «néant » pour donner l'équivalent de ce que Flaubert conçoit comme «l'agonie » à laquelle il consacre toute une esthétique. Dans «Yasmina », «La félicité de l'heure » (102) ou flânerie est cette soustraction faite au temps, pour fuir la réalité contraignante de deux amants qui ne peuvent s'unir dans le monde où ils vivent, fait d'adversités. Pour Eberhardt, cet Orient vaste offre la possibilité d'un lien entre ses concepts libertaires et des notions renouvelées qui nous préoccupent aujourd'hui comme le moment présent, la pleine conscience, l'échappatoire spirituelle au temps. Chez Eberhardt, la flânerie orientale introduit une subversion dans la flânerie née dans la ville. Sa façon de flâner introduit la pluralité et appelle à transgresser les limites, les étirant jusqu'en Orient, en ville ou dans le désert. Eberhardt traduit sa rébellion par le nomadisme : « Nomade j'étais quand, toute petite, je rêvais en regardant la route, la blanche route attirante qui s'en allait, sous le soleil qui me semblait plus éclatant, toute droite vers l'inconnu charmeur [...], nomade je resterai toute ma vie, amoureuse des horizons changeants, des lointains encore inexplorés, car tout voyage, même dans les contrées les plus fréquentées et les plus connues, est une exploration » (t. 1, Journaliers, 231). Son choix de partir pour le désert et de se détacher des liens sociaux porte en soi la quintessence de l'idée de résistance. Dans l'acte de résistance, il $\mathrm{y}$ a des risques à prendre, des dangers à affronter et les biographes d'Eberhardt le soulignent : 
«Sa dérive, manière de contestation paresseuse mais provocante en cette fin de siècle colonial, l'expose à tous les dangers » (Delacour et Huleu 12). La contestation paresseuse n'est-elle pas une forme larvaire de la rébellion?

\section{Le Spleen oriental}

Pour Eberhardt, l'Orient, cet ailleurs rêvé, est le centre de la flânerie et elle s'y rend. Elle se positionne contre ce qu'elle appelle l'envahissement de l'Occident, ainsi cette Europe en pleine expansion économique et industrielle qu'elle fuit. La flânerie est motivée par l'envie d'échapper aux contraintes de la vie programmée par le pragmatisme et régie par l'utilitarisme. En ce sens, elle est une action de résistance ou de rébellion mais elle est aussi élévation de l'esprit. Nietzsche, l'un des défenseurs de la lenteur, écrit dans Le gai savoir: «La beauté sauvage de l'irrationalité poétique vous réfute, utilitaristes! Vouloir précisément se libérer enfin de l'utile - Voilà ce qui a élevé l'homme, voilà ce qui lui a inspiré la moralité et l'art !» (139). La flânerie en Orient constitue pour Eberhardt l'expression du refus des valeurs pragmatiques, utilitaristes et matérialistes qui régissent la vie moderne en Europe au dix-neuvième siècle. Flâner, c'est consommer de l'énergie, consumer le temps, par opposition à la valeur matérialiste de produire (en valeur monétaire).

D'un autre point de vue, comme chez Charles Baudelaire dont la flânerie est motivée par le spleen qui l'anime, c'est un chagrin vivace qui pousse Eberhardt à vagabonder loin des confins des villes. Elle porte en elle un spleen oriental qu'elle exprime ainsi dans sa réflexion Vers les horizons bleus : "Louange à la souffrance du cœur ! Louange à la mort fécondatrice des âmes endeuillées ! Louange au tombeau silencieux qui est non seulement la porte de l'éternité pour ceux qui s'en vont, mais encore celle du salut pour les âmes élues qui savent se pencher sur ses profondeurs mystérieuses! Louange à la tristesse et à la mélancolie, ces divines inspiratrices !» (t. 1, 67). Lors de son vagabondage, elle fait des rencontres fortuites qui la touchent profondément, comme le chanteur inconnu accompagné d'une flûte de roseau qui, au clair de la lune, le soir près de sa demeure à Tunis, chante une mélodie qu'elle comprend et qui parle à son âme. Elle retranscrit cette scène, qui n'aurait jamais eu lieu dans Paris au dix-neuvième siècle, mais qu'elle rencontre pendant ses «Heures de Tunis »:

Il était parfaitement beau et avait le teint mat et blanc des citadins arabes [...] mais son visage était empreint d'une tristesse profonde.

Il s'asseyait là, toujours à la même place, et, le regard perdu dans l'infini bleu de la nuit, il chantait, sur des airs d'autrefois éclos sous le ciel d'Andalousie, des cantilènes suaves. Lentement, doucement, sa voix montait dans le silence, comme une plainte ou une incantation... 
Il semblait surtout préférer ce chant, le plus doux et le plus triste de tous : « Le chagrin vivace étreint mon âme, comme la nuit étreint les choses et les efface. La douleur étreint mon cœur et le remplit d'angoisse, comme le tombeau étreint les cœurs et les anéantit. À ma tristesse, il n'est pas de remède, sauf la mort sans retour... Mais, si plus tard, mon âme se réveille pour une autre vie, fût-ce celle d'Eden, ma tristesse renaîtra en elle ».

Quelle était cette tristesse incurable, dont l'inconnu chantait la puissance ? Le chanteur singulier ne le dit jamais. Mais sa voix était pure et modulée, et jamais aucune autre ne m'avait livré aussi pleinement le charme secret et indéfinissable de cette musique arabe de jadis, qui enchanta, avant la mienne, bien d'autres âmes tristes. (30-31)

Eberhardt nous livre un spleen oriental en pleine nuit dans une rue de Tunis. Chez elle, des scènes authentiques de la vie de tous les jours deviennent, grâce à son style sobre et raffiné, romanesques.

\section{Conclusion}

Le vagabondage est l'expression d'une position d'ubiquité sociale qui traduit pour Eberhardt l'essence même de la liberté: «Être étranger et chez soi partout» («Vagabondages, 27). Cette ubiquité lui permet de se prononcer sur les idées conçues dans le monde occidental comme étant indiscutables, par exemple le récit de la Genèse qui place la responsabilité de la tentation par la chair sur la femme. Son choix de l'espace amorphe du désert peut être la réflexion de ses choix de vie marginaux et quelque peu anarchistes. En s'opposant à tout ce qui constitue l'idéologie occidentale et en vivant sa philosophie par le vagabondage, Eberhardt rompt avec l'idéologie en tant qu'espace fermé qui entrave la liberté des esprits anarchistes. Philippe Hamon, dans Texte et idéologie, souligne que «l'idéologie [...] s'oppose donc, par sa logique, à l'espace amorphe de l'anarchie, où n'existent ni contraintes ni interdictions, où tout est à la fois permis et facultatif » (103). Il semblerait que cet espace amorphe de l'anarchie en laquelle Eberhardt écrit et croit, est l'espace de son identité Mais quel est le lien entre son choix du soufisme et sa vision anarchiste des choses ? Selon les critiques et les biographes d'Eberhardt, les principes de l'anarchisme ont jalonné l'enfance et la jeunesse de l'auteure, sous la tutelle de Trophimowsky, disciple de Bakounine. Une forme d'anarchisme continue à se lire à travers sa vie et dans ses œuvres. C'est ce qu'affirme par exemple Simone Rezzoug :

Isabelle Eberhardt fut élevée dans ce contexte. L'ignorer, c'est risquer un contresens sur les idées qu'elle affiche dans son œuvre, sa haine de la civilisation, ses proclamations d'indépendance. Conformément aux idées anarchistes de l'époque, elle fut élevée par son « père » « comme un garçon », aucune distinction ne devant être faite entre les sexes selon les préceptes 
libertaires. Il lui apprend à scier du bois, à monter à cheval; il lui enseigne le russe, l'allemand, le latin et lui fait donner des cours d'arabe. Cette conception de l'éducation est conforme aux impératifs libertaires : les anarchistes ont été très tôt passionnés par les problèmes de l'enseignement et de la transmission d'une morale tout humaine se fondant " sur le mépris de l'autorité et sur le respect de la liberté et de l'humanité ». (21)

Dans ses récits et journaux par exemple, Eberhardt évoque des figures de femmes maghrébines en marge de la société qui pourraient être considérées comme des figures anarchistes musulmanes. Cependant, aucune étude à ce jour n'a encore exploré le lien entre le mode de vie d'Eberhardt, sa philosophie et ses idées anarchistes.

\section{Ouvrages cités}

Abdel-Jaouad, Hédi. «Isabelle Eberhardt : Portrait of the Artist as a Young Nomad ». Yale French Studies 83 (1993). 93-117.

Behdad, Ali et Laura Rice. Belated Travelers: Orientalism in the Age of Colonial Dissolution. Durham : Duke UP, 1994.

Chaulet Achour, Christiane. «Isabelle Eberhardt (1877-1904) : une identité dans l'altérité ».

Diacritik, Livres et portraits, 2016, <https://diacritik.com/2016/12/23/isabelleeberhardt-1877-1904-une-identite-dans-lalterite/ >, consulté le 15 novembre 2018.

Chomsky, Noam. Instinct de liberté : anarchisme et socialisme. Montréal : Comeau et Nadeau, 2001.

Delacour, Marie-Odile et Jean-René Huleu. «Heures de Tunis ». Oeuvres complètes, t. 1. Paris : Grasset, 1988.

Eberhardt, Isabelle. Euvres complètes, t. I-II. Paris : Grasset, 1988.

Hamon, Philippe. Texte et idéologie : valeurs, hiérarchies et évaluations dans l'œuvre littéraire. Paris : PUF, 1984.

Nesci, Catherine. Le flâneur et les flâneuses : les femmes et la ville à l'époque romantique. Grenoble : Ellug, 2007.

Nietzsche, Friedrich. Le Gai Savoir par-delà bien et mal. Tr. Patrick Wotling. Paris : Flammarion, coll. «Le Monde de La Philosophie », 2008.

Rezzoug, Simone. Isabelle Eberhardt. Alger : OPU, « coll. Classiques maghrébins », 1985.

Shindler, Patrick. Arthur Rimbaud : l'anarchiste inachevé. Paris : Monde Libertaire, 2001. 